International Journal on Intelligent Electronic Systems, Vol. 4, No.2, July 2010

\title{
AVERAGE-HALF-FACE FOR FACE RECOGNITION USING PCA \& WAVELET TRANSFORM
}

\author{
Sumathi S. ${ }^{1}$, RaniHema Malini R. ${ }^{2}$. \\ ${ }^{1}$ Research Scholar, Sathyabama University, Chennai, Tamilnadu, India. \\ ${ }^{2}$ Dept of E\&I, St.Peter's University, Chennai, Tamil Nadu, India. \\ Email: '1 sumathi_ba@rediff mail.com, ${ }^{2}$ ranihema@yahoo.com
}

\begin{abstract}
With the tremendous development in technology, security system has also reached its peak in development. Biometrics has played an important role in security system in the past decades. As the most natural and friendly identification method, automatic face recognition has become the important part of the next generation computing technology. 3D face recognition methods are able to overcome the problems resulting from illumination, expression or pose variations in $2 \mathrm{D}$ face recognition. Many face recognition algorithms have been developed and each has its strengths. Each shares a common element which is that they all input a full face image into the algorithm. They need more memory space and take large computation time. Utilize the symmetry of the face for face recognition, select average half-face for research. The consequence of this discovery may result in substantial savings in storage and computation time. We have good response on the average half face image using wavelet transformation; reduce the computation time for each image is one third of the full image. And also we determined the memory storage is reduced the half of the full image. We save memory space for each image is 60KB. Applying the average-half-face to additional algorithms and databases (2D\&3D) and analyzing the effect of illumination, facial expressions and occlusions. A face recognition method that is able to recognize faces at various angles is proposed.
\end{abstract}

Keywords: Face recognition, Biometrics, Average-half-face, Wavelet transform, computation time, memory

\section{INTRODUCTION}

Biometric are used to identify people based on their biological traits, authentication is the process of using a unique physical or behavioral trait as a method to confirm the identity and determine the access profile of a person. Automatic recognition of people has received much attention during the recent years due to its many applications in different fields such as law enforcement, security applications or video indexing. Face recognition is an important and very challenging technique to automatic people recognition. It has the potential to provide significant benefits to society.

Face recognition is one of the significant technologies in biometrics due to its robust application in security systems, identification of criminals, and assistance with speech recognition system, surveillance, Closed Circuit Television (CCTD) and user identification. Facial recognition offers several advantages. Since faces can be captured from some distance away, facial recognition can be done without any physical contact. Unlike human beings who have the excellent capability to recognize different patterns and faces, machines are still lacking of this aptitude due to its variations in illuminations, complex backgrounds, visual angles, facial expressions and therefore, face recognition has become a complex and challenging task for researchers, scientists and physicists over the last two decades.

As a biometric, face image is the least intrusive, but several challenges remain in improving the accuracy of face recognition under illumination changes, variations in pose, occlusions (including self-occlusion), image resolution and other such difficulties. Many face recognition algorithms have been developed and each has its strengths. Each shares a common element which is that they all input a full face image into the algorithm. However, none of the methods currently exploit the inherent symmetry of the face for recognition [1].

In this paper we demonstrate the effectiveness of using the average-half-face as an input to face recognition algorithms for an increase in accuracy and potential decrease in storage and computation time [2]. The average- half-face is a transformation method that attempts to preserve the bilateral symmetry that is present in the face. It has been shown to increase the accuracy of face recognition with eigenfaces applied to 
3D face images. In this technique, first the nose tip is automatically located on the range image, and then the range data from hexagonal region of interest around this landmark is decomposed using wavelet levels. The dimensionality of the extracted coefficients at each resolution level is reduced using PCA [3]. Almost all face recognition research in the early days was focused on using portrait images of the face.

\section{AVERAGE-HALF-FACE}

It is well-known that the "face is roughly symmetrical" [4] and use the idea of face symmetry to solve the problem of illumination in face recognition using Symmetric Shape-from-Shading. Introduce the notion of 'Half-faces' (in the sense of exactly one half of the face) to assist in computing a similarity measure between faces using images that have non-uniform illumination. In face recognition, the use of the bilateral symmetry of the face has been limited to extracting facial pro?les for recognition [5] when applied to face images of $2 \mathrm{D}$ or $3 \mathrm{D}$ [2] this amounts to two steps. First, the image is centered about the nose of the face to represent the data as symmetric as possible. When we speak of the data being symmetric, We mean that two spatial halves of the data are similar, not that the matrix of the data itself is symmetric. Next, the image is divided into two symmetric halves and they are averaged together (reversing the columns of one of the halves first).
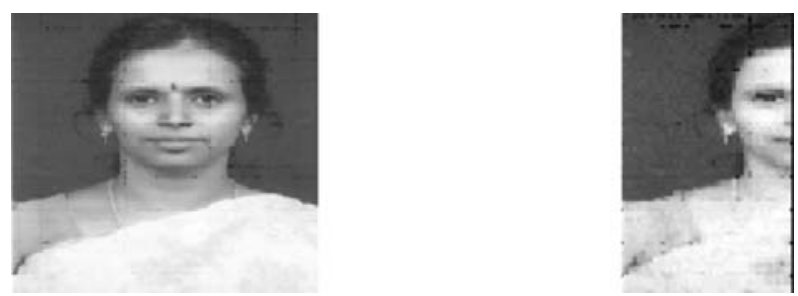

Fig. 1 (a) 2D full face image; (b) average-half-face;
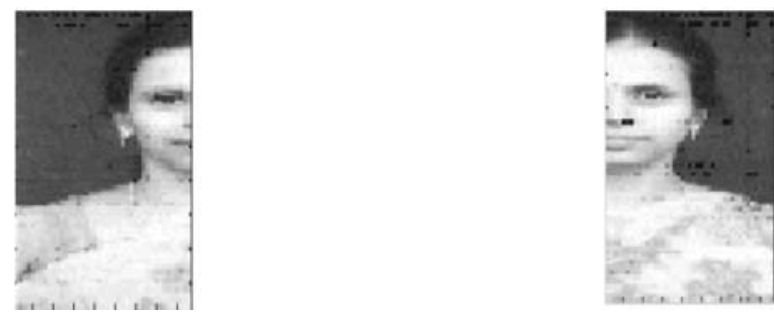

(c) left half-face; and (d) right half-face.

\section{FACE RECOGNITION ALGORITHM}

\section{A. Principal Component Analysis}

Principal Component analysis is standard technique used in statistical pattern recognition and signal processing for data dimensionality reduction and feature extraction. PCA is one of the most successful techniques that have been in image recognition and compression. The purpose of PCA is to reduce the dimensionality of the feature space, which is needed to describe the data economically. As the pattern often contains redundant information, mapping it to a feature vector can get rid of this redundancy and yet preserve most of the intrinsic information content of the pattern. These extracted features have great role in distinguishing input patterns [6]. Every test image can be transformed to low dimensional feature vector to be projected on to the eigenface space which was obtained from the training set. This feature vector can then be compared with the set of feature vectors obtained from the training set. The face classifier can use different distance measure such Euclidean distance or cosine distance. The PCA algorithm can be detailed as follows. Let $X=\left(x_{1}, x_{2}, \ldots . . x_{k}, x_{N}\right)$ to represent the $d \times N$ data matrix, each $x_{k}$ is a $1 D$ - vector of size $d$ containing the reordered version of the $2 \mathrm{D}$ face image [7]. $N$ represents the number of face images in the training set. PCA is simply a linear transformation from original image vector to projection feature vector such that,

$$
Z=W^{T} X
$$

Where $Z$ is the $m \times N$ feature vector matrix, $m$ is the dimension of the feature vector, and transformation matrix $W$ is an $d \times m$ transformation matrix whose columns are the eigenvectors corresponding to the $m$ largest eigenvalues computed using formula

$$
\lambda e_{i}=S e_{i}
$$

where $S$ is the total scatter matrix, which uses the mean image of all training samples and define as

$$
S=\stackrel{N}{\Sigma}\left(x_{1}-\mu\right)\left(x_{i}-\mu\right)^{T}
$$


Sumathi et al: Average-half-face for Face Recognition

$$
\mu=1 / N \sum_{i=1}^{N} x_{i}
$$

After applying linear transformation WT, the scatter of the transformed feature vectors $Z=\left(z_{1}, z_{2}, \ldots, z_{N}\right)$ is $W^{\top} S W$. the sub space of PCA is spanned by a set of vectors

$W_{\text {opt }}=\left[w_{1}, w_{2}, \ldots, w_{M}\right]$, which is a set of eigenvectors ${ }^{2}$ of corresponding to the largest $m$ eigenvalues. The Projection [5]

$$
\mathrm{W}_{\mathrm{opt}}=\arg \max _{\mathrm{W}}\left[\mathrm{W}^{\top} \mathrm{SW}\right]
$$

Classification is performed by comparing the feature vectors of the training face images with the feature vector of the input / test face image. The comparison can be based on the Euclidean distance or cosine distance between the face classes and the input / test face image. Each face in the training set is transformed into the face space and its components are stored in memory. This space has to be populated with these known faces.

\section{B. Wavelet Transform}

Wavelet transformations are a method of representing signals across space and frequency. The signal is divided across several layers of division in space and frequency and then analyzed. The goal is to determine which space/frequency bands contain the most information about an image's unique features, both the parts that define an image as a particular type (fingerprint, face, etc.) and those parts which aid in classification between different images of the same type. One type of discrete wavelet transform (DWT) is the orthogonal DWT. The orthogonal DWT projects an image onto a set of orthogonal column vectors to break the image down into coarse and fine features. Also, the coefficients of wavelet decomposition provide information that is independent of the original image resolution. Thus, a wavelet based scheme allows us to easily compare images of different resolutions. And finally, wavelet decompositions are fast and easy to compute, requiring linear time in the size of the image and very little code.

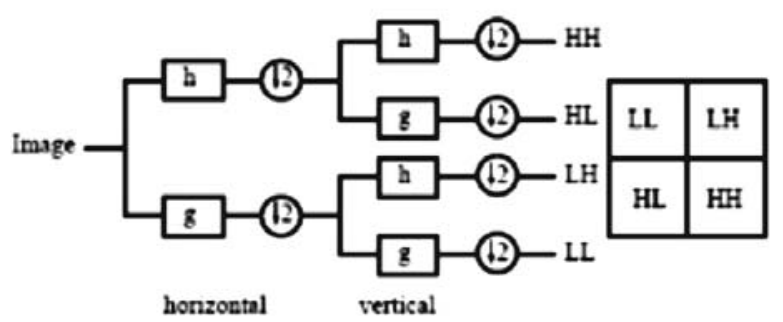

Fig. 2 Block diagram of Discrete Wavelet Transformation

Typical two-level full wavelet decomposition is shown above. Each of the subspaces is obtained by taking the original input and filtering it with a combination of high-pass and low-pass filters, designed to maximize the amount of information obtained within each subspace. This decomposition can be repeated for n-levels. The image can later be reconstructed from these subspaces. By removing subspaces that contain comparatively low amounts of information from a reconstruction, we can achieve an image that is nearly as good as the original but takes less space to store. This can be useful if we are storing a large number of similar images.

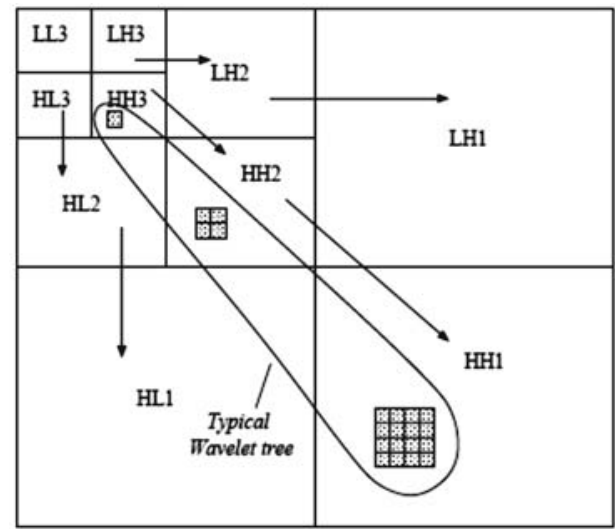

Fig. 3. Space-frequency structure of wavelet transforms

The Discrete Wavelet Transforms (DWT) is being increasingly used for image coding. In particular, biorthogonal symmetric wavelets manifested remarkable abilities in still image compression. In this we present a new family of biorthogonal wavelet transforms, which are proven to be efficient for image compression. DWT has traditionally been implemented by convolution. Such an implementation demands both a large number of computations and a large storage features that are not desirable for high speed or low power applications. 
But the lifting based scheme that often requires far fewer computations has been proposed for the DWT. The main feature of the lifting based DWT scheme is to break up the high pass and low pass filters into a sequence of upper and lower triangular matrices and convert the filter implementation into banded matrix multiplications.
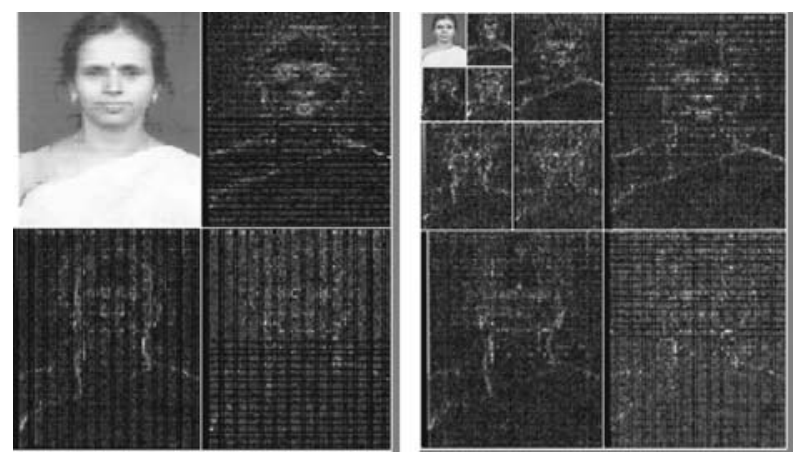

Fig. 4 Face image in wavelet subbands

(a) 1 - level wavelet decomposition

(b) 3 - level wavelet decomposition

Discrete wavelet transform is used to decompose the face image into a multiresolution representation in order to keep the least coefficients possible without losing useful image information. Demonstrates the decomposition process by applying two- dimensional wavelet transform of a face image in level depicts three levels wavelet decomposition by applying wavelet transform on the low-frequency band sequentially. It can be convenient for solving the error rate and false alarm in the process of recognition. This paper presents a three dimensional face recognition methods based on the geometry, which is based on lie: Even if a person's facial features are rather ambiguous, as long as it has relative location and size, face is enough to be identified.

To join curve distance as feature, form feature vectors of Euclidean distance, curve distance, angle and volume, then compare with the face model of template set and complete face recognition. Our scheme is based on machine learning which combined wavelet transform. Regularly sequential searching and recognizing engendered excessive calculation and cost long time [8].

\section{3D FACE RECOGNITION APPROACH}

Wavelet transformation uses in three dimensional geometrical features to rcognize the face directly, that is to say the facial sample database store three dimensional model, and to be recognized face is a also three - dimensional facial model [9].

Recognition algorithms as follows:

1. Capturing to be recognized human faces.

2. Feature extraction.

3. Measuring three - dimensional geometric is to obtain the geometric characteristics.

4. Needing similarity judgment between vectors are to be recognized facial feature and the facial features in the samples. Similarity is calculated as follows:

$$
S=\sqrt{\sum_{i=1}^{14}\left(F_{i}-F_{i}\right) W_{1}}
$$

Where $F_{i}$ is $i$-eigenvector of the samples to be detected, $F_{i}^{\prime}$ is $i$ - eigenvector of the samples in the samples. $w_{i}$ is the weight of the i-eigenvector; the weight is determined by the experiential formula and the importance of facial features.We set the similarity threshold $\theta$ to compare samples to be recognized with the ones in the samples. If $S<\theta$ the two samples is similar, otherwise. Lastly, the $3 \mathrm{D}$ face recognition is completed [12].

\section{ALGORITHM VERIFICATION \& IMPLEMENTATION}

The parameters for each algorithm were kept constant between experiments to maintain a fair comparison of each algorithm's performance on the average-half-face and the full face. Also, the images were centered for both the average-half-face and full face recognition results. Each of the numbers in the table represents the rank-1 accuracy rate for recognition, meaning that we report only the accuracy of the closest match of the test data to a corresponding training sample [1]. We first performed PCA on the entire 3D face data (109 images) of full face images and on their corresponding average - half-face images to discover the amount of cumulative variance captured for each eigenvector. We did this on the full face and on the average-half-face as shown in Figure 6. Table 
2 lists a few examples of the number of eigenvectors needed for both the full face.

The average- half-face in order to capture the same amount of cumulative variance in the data. In order to capture the same amount of variance in the data, fewer eigenvectors are required for the average-half- face than for full faces.

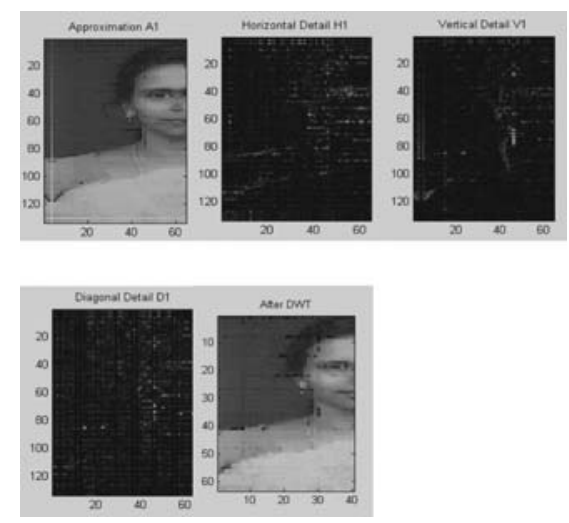

Fig. 5 Output images of DWT AHF

It is crucial to recall when studying that table that the purpose of these experiments is to compare the full face to the average-half-face for recognition, not to compare the accuracy of the algorithms themselves. We can clearly see that the average-half-face outperforms the full face in every method for the Yale Face database and the 3D face database [11].

Table 1. Rank-1 accuracy results using the full face (Full), Average-half-face (HF) and Wavelet Transformation (WT).

\begin{tabular}{|c|c|c|c|c|c|c|}
\hline \multirow{3}{*}{$\begin{array}{l}\text { Data } \\
\text { base }\end{array}$} & \multicolumn{2}{|c|}{ A } & \multicolumn{2}{|c|}{ B } & \multicolumn{2}{|c|}{ C } \\
\hline & \multicolumn{2}{|c|}{ Yale } & \multicolumn{2}{|c|}{ AR } & \multicolumn{2}{|c|}{ 3D } \\
\hline & Full & HF & Full & HF & Full & HF \\
\hline PCA & 77.8 & 86.7 & 49.4 & 52.3 & 72.8 & 80.4 \\
\hline WT & 80 & 93.5 & 50.8 & 65.2 & 75.3 & 89.6 \\
\hline
\end{tabular}

The next set of experiments performed involved different types of preprocessing applied to the original data. We preprocessed each image to produce an average-half-face, a left half-face (right half of the image, which contains the left half-face) and a right half-face for our experiments. For each of the experiments, the reported accuracy is the rank 2 accuracy, meaning that the correct classification is recorded when the correct match in the gallery is one of the two nearest images in the subspace [11].

\section{DISCUSSIONS}

In accordance with the standard practice for evaluating face recognition systems, we divided the 3D data set into three disjoint sets; train, gallery and probe sets. For the training set, we used 109 images total of 12 different subjects with (smiling and neutral) facial expressions. For the gallery set, we used one image with a neutral expression for 84 different subjects. The remaining images of the 25 subjects were used as the probe set. In the probe set, the number of range images in the probe set per subject varied from 1 to 55. We used the minimum Euclidean distance in the projected subspace to classify the projected probe images to the nearest projected gallery image [1].

Every Eigen faces are compare with full image face along the Average right and Left image. Each an every $2 \mathrm{D}$ image similarities are matched with $3 \mathrm{D}$ image varies along their facial expressions. In this matches done using the 3-level decomposition of an image. System is response of an image very quickly and easier to computation the Average Half face.

In our 3D face recognition experiments, the accuracy of the eigenfaces recognition when using only one half of the face (either the right or left half-face) is less than, or equivalent to, using the full face. This leads us to the conclusion that we are missing valuable information to assist in face recognition when we only consider one half of the face. It is interesting, however, that using the left half-face alone produces comparable accuracy to using the full face. Randomly choosing half of the face also performs the same or worse than using the full face. However, it is clear that in our experiments the average-half-face produces higher accuracy than the original full face data. This gain in accuracy is clearly not coming from simply using one half of the face versus the other, or even using both halves of the face simultaneously. The conclusion we draw is that the gain in accuracy has origins in the average of the two sides of the face, which is a very interesting finding. At this point in the investigation, it is not clear what other factors are behind this increase in accuracy. 
Table. 2 Summary of the resource usage and executions times for varying image sizes

\begin{tabular}{|l|c|c|c|}
\hline \multicolumn{1}{|c|}{ Section } & $\begin{array}{c}\text { Capture } \\
\text { Average } \\
\text { Time (Sec) }\end{array}$ & $\begin{array}{c}\text { Memory } \\
\text { Space (KB) }\end{array}$ & $\begin{array}{c}\text { Computation } \\
\text { Time (Sec) }\end{array}$ \\
\hline Full image & 5.75 & 80 & 386 \\
\hline Image resize & 1.05 & 50 & 255 \\
\hline Half-Face & 0.35 & 25 & 127 \\
\hline WT-Face & 0.25 & 20 & 100 \\
\hline
\end{tabular}

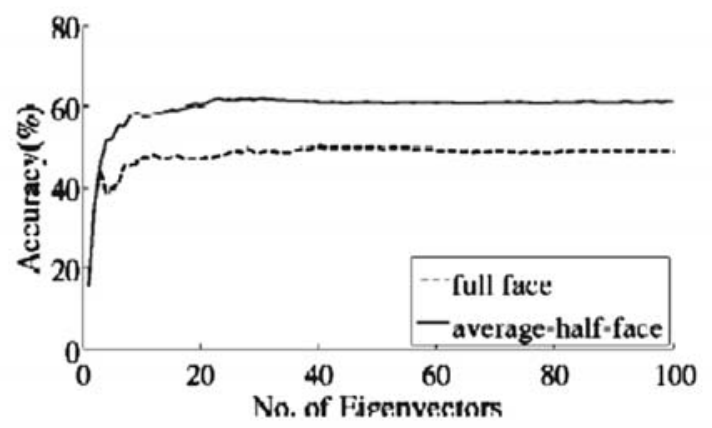

Fig. 6 Rank 2 accuracy using the average-half-face.

It is abundantly apparent from Table 1 that regardless of the algorithm using average-half-face with the Yale Face database and the 3D database produces an equal or higher accuracy rate than when using the original full face. This is not the case for every method when using the Yale Face database [11]. We have good response on the average half face image using wavelet transformation; reduce the computation time for each image is one third of the full image. And also we determined the memory storage is reduced the half of the full image. We are saved each image memory space is $60 \mathrm{~KB}$.

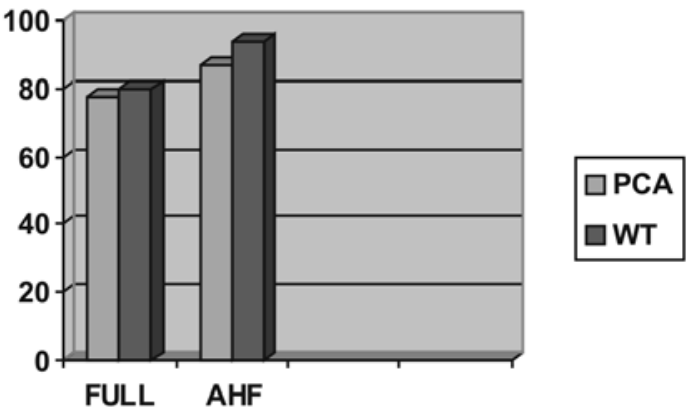

Fig. 7. Accuracy of Full Face and Average-Half-Face on Yale Face database (A).
For instance, when using the AR Face database, the rank-1 recognition rates of the PCA and Wavelet transformation methods are notably better when using the full face versus using the average-half-face. For the Yale Face database, the eigenfaces (PCA) methods perform $6-13 \%$ better with the average-half-face than with the full face. All other methods with the Yale Face database are comparable, but usually have better results with the average-half-face. The 3D database gives consistently better results when using the average-half-face with a maximum accuracy increase of around $8 \%$ with the eigenfaces (PCA) method. Therefore, with a simple computation step, the accuracy of the majority of the algorithms tested was improved. We believe that this gain in accuracy has it's origin in the averaging operation, which produces a new face that contains a set of features that are more discriminatory that those of the full face [12].

\section{CONCLUSIONS}

Using Wavelet transformation with simple calculation of the average-half-face computed from the full face, the rank-1 accuracy of recognition is improved, regardless of database or algorithm utilized. We applied the average-half-face to facial feature extraction methods, such as used discrete wavelets transforms. Our approach increased the face recognition accuracy and more robust to effect of illumination, facial expressions, occlusions, etc. In multi-target gallery, our clustering scheme can be more quickly to identify a probe. A set of novel features for $3 \mathrm{D}$ face recognition that combines wavelet transformation with PCA. The range data from a triangle region of interest is decomposed using wavelet transformation. The dimensionality of the extracted coefficients at each resolution level is reduced using principal component analysis. Experimental results on a database of 109 range images showed that by using only 35 PCA coefficients in a simple nearest neighbor classifier, recognition rates as high as $94.17 \%$ are achieved.

Further more; we think there are 3 points needed be researched deeply: 1) Combine with the other sub band coefficients of wavelet decomposition or some local place analysis with WT to improve recognition ability is needed. 2) Concentrate on View points influence on face recognition. When face rotates exceed 15 degree, recognition accuracy decreases sharply. Multi view point WT sub band or 3-D based 
face recognition is another valuable suggestion for discussion. 3) More clustering algorithms should be involved and compared with think of the features of human face.

\section{REFERENCES}

[1] Harguess J., Gupta S., and Aggarwal J.K.(2009). 'A case for the Average - Half- face in $2 \mathrm{D}$ and $3 \mathrm{D}$ for face recognition',IEEE Transactions on Digital Object Identifier, CVPR,10. 1109. pp.7-12.

[2] Harguess J. Gupta S., and Aggarwal J.K., 2008 '3D face recognition with the average-half-face' International Conference on Pattern Recognition ICPR.

[3] Sina Jahanbin, Hyohoon chor, Alan C.Bovik, Kenneth R.Casleman.,(2007), 'Three Dimensional Face Recognition using Wavelet Decomposition of Range Images', IEEE Transaction on Computer Vision, ICIP 2007,pp.145-148.

[4] Shakhnarovich G., Moghaddam B., (2004) 'Face recognition in subspaces', In; S.Z. Li, A.K. Jain (E ds.), Handbook of Face Recognition, Springer.pp. 141- 168.

[5] Pan G. and Wu Z. (2005), ' 3D face recognition from range data'. Int. J. Image Graphics, 5(3):pp 573-594.

[6] Alaa Eleyan, Hasan Demirel and Huseyin Ozkaramanli.,(2008). ' Face Recognition using Dual Tree Wavelet Transform': IEEE Symposium on Signal
Processing and Information Technology, (ISSPIT 2008). pp. 7-11.

[7] Kyungnam Kim. (1996), 'Face Recognition using Principle component Analysis', International Conference on Computer Vision and Pattern Recognition, pp.586-591.

[8] Abbas Bigdeli, Colin Sim,Morteza-Abhari,Barin C.Lovell. (2007), 'Face Recongnition on Embedded Systems', IEEE International on Embedded software and sysems,ICESS,pp. 295-308 .

[9] Yu Song, Wenhong Wang, Yanyan Chen, (2009), 'Research on 3D Face Recognition Algorithm',First International workshop on Education Technology and computer Science10.1109,pp.47-50.

[10] Bing Luo, Yun Zhang, Yun-Hong Pan,(2005), ' Face Recognition Based on Wavelet Transform and SVM', IEEE International Conference on information Acquisition/ICIA.: pp.373-377 .

[11] Yale Univ. Face DB, 2002. http://cvc.yale.edu/projects/yale faces/yalefaces.html.

[12] Neo Han Foon, Ying-Han Pang, Andrew Teoh Ben Jin,David Ngo Chek Ling,(2004), ' An Efficient Method for Human Face Recognition Using Wavelet Transform and Zernike Moments', IEEE International Conference on Computer Graphics, Imaging and Visualization (CGIV'04). 